\title{
A simplified method of evaluating restraint and reinforcement effect on concrete restrained stress
}

\author{
Jianda Xin $^{1, a}$ and Dahai Huang ${ }^{1, b}$ \\ ${ }^{1}$ Department of airport and pavement engineering, Beihang University, Beijing, China \\ axinjianda@buaa.edu.cn \\ bhuangdh@tsinghua.edu.cn
}

Keywords: reinforced concrete, restraint degree, creep, age-adjusted effective modulus method Abstract: In this paper, effects of restraint degree and reinforcement on concrete restrained stress have been analyzed based on the age-adjusted effective modulus method (AAEMM). Based on principle of deformation compatibility and force equilibrium, formulations relating to the stress and strain of reinforcement and concrete have been derived; meanwhile, the calculating algorithm is programmed with the Matlab software andrun throughout the analysis procedures. Results show that more concrete temperature stress can be transferred to reinforcement with slower temperature variationand lower restraint degree during long-term loading periods. The relationship between restraint degree and concrete restrained stress reduction ratio has been developed.

\section{Introduction}

Numerous deformations are generated during the hardening process of concrete: autogeneous deformation caused by self-desiccation, temperature deformation caused by hydration heat and drying deformation caused by moisture loss under drying condition. The deformation of concrete members is often restricted by the adjoining structures or soil foundations and the restrained stress is generated [1]. Creep is another time-dependent behavior of concrete and the crack possibility of concrete considering creep effect can be greatly lowered [2].

For reinforced concrete structures under the short-term loading, the usage of reinforcement is not to prevent concrete from cracking [3], but to limit the deflection and crack width of reinforced concrete members [4,5]. Meanwhile, for reinforced concrete structures considering hydration heat generation process, the restrained stress caused by temperature variation and external restraint is much greater than that of stress caused by external loads.

In this paper, a simplified method for evaluating effect of restraint degree and reinforcement on concrete restrained stress has been established and the relationship between restraint degree and concrete restrainedstress reduction has been quantitatively assessed.

\section{Modelling}

Restraint degree.As shown in Fig.1, the free deformation of uniaxial concrete member is $\Delta I_{f}$, the actual deformation of uniaxial concrete member under a certain restraint is $\Delta l_{r}$, and then the restraint degree $\gamma_{R}$ can be defined as

$$
\gamma_{R}=\frac{\Delta l_{f}-\Delta l_{r}}{\Delta l_{f}} .
$$

The numerator represents the restrained deformation and the restraint degree is denoted by the ratio of this deformation difference to the free deformation.

Basic assumption.(1) The elastic creep theory is available [6]

$$
\varepsilon_{\text {total }}(t)=\sigma(\tau)\left[\frac{1}{E(\tau)}+C(t, \tau)\right]+\int_{\tau}^{t}\left[\frac{1}{E(\tau)}+C(t, \tau)\right] d \sigma(\tau) .
$$

where $E(\tau)$ is the concrete elastic modulus at loading age of $\tau, E(t)$ the concrete elastic modulus at age of $t, C(t, \tau)$ the specific creep of concrete;

(2)The deformation compatibility between reinforcement and concrete is valid; 
(3)No consideration of concrete crack.

Modelling process. The following stress-strain relationship of concrete is established based on the age-adjusted effective modulus method (AAEMM)

$$
\varepsilon_{\text {total }}(t)=\frac{\sigma_{0}(\tau)}{E_{c}(\tau)}[1+\varphi(t, \tau)]+\frac{\sigma(t)-\sigma_{0}(\tau)}{E_{c}(\tau)}[1+\chi(t, \tau) \varphi(t, \tau)]+\varepsilon^{0}(t, \tau) \cdot
$$

where $\varphi(t, \tau)$ is the creep coefficient of concrete; $\chi(t, \tau)$ is the ageing coefficient of concrete; $\varepsilon^{0}(t, \tau)$ is the stress-independent deformation of concrete.

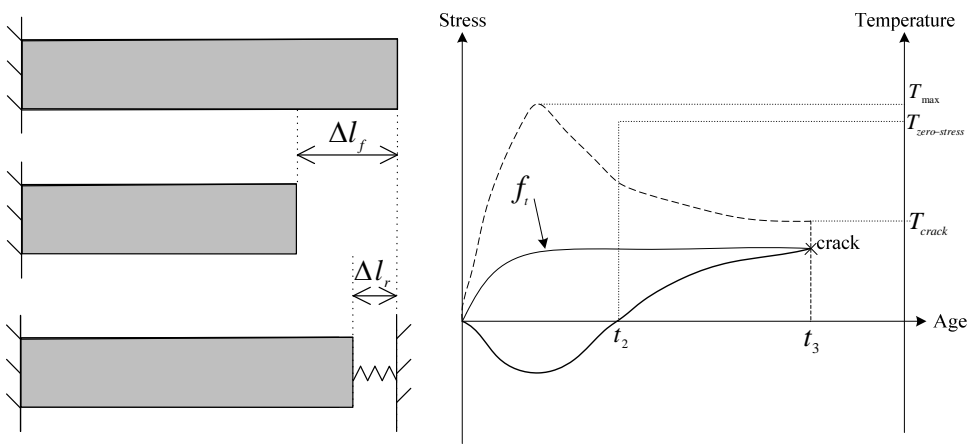

Fig.1. Deformation of concrete member withaFig.2. Evolution of temperature and stressrestraint degree

The restraint stress of concrete member at age $t$ is

$$
\begin{array}{r}
\sigma(t)=\int_{t} R(t, \tau) \cdot d \varepsilon(t)+\sigma^{f i x}(t) . \\
\sigma^{f i x}(t)=\int_{t} R(t, \tau) \cdot d \varepsilon^{0}(t) .
\end{array}
$$

where $R(t, \tau)$ is the relaxation modulus of concrete; $\varepsilon(t)$ is the actual deformation of concrete; $\varepsilon^{0}(t)$ is the free deformation of concrete; $\sigma^{\text {fix }}(t)$ is the restrained stress when concrete member is totally restrained.

Then, one can obtain that

$$
\gamma_{R}=\frac{\sigma(t)}{\sigma^{f i x}(t)}=\frac{\int_{t} R(t, \tau) \cdot d \varepsilon^{0}(t)-\int_{t} R(t, \tau) \cdot d \varepsilon(t)}{\int_{t} R(t, \tau) \cdot d \varepsilon^{0}(t)}
$$

The creep deformation then can be expressed as

$$
\varepsilon^{\text {creep }}(t)=\gamma_{R} \cdot \varepsilon^{0}(t)\left[1-\frac{E_{c}(\tau)}{E_{c}(t)(1+\chi(t, \tau) \varphi(t, \tau))}\right] \cdot
$$

Eq.7 shows the relationship between restraint degree and concrete creep. As shown in Fig.2, once the second zero stress age $t_{2}$ and cracking time $t_{3}$ are known, the creep deformation of concrete with different restraint degrees can be quantitatively calculated. The concrete stress and strain calculation method in [7] isadopted andprogrammed with the Matlab software, then the second zero stress age $t_{2}$ and cracking age $t_{3}$ can be obtained.

Based on Eq.3, the strain increment of reinforced concrete member can be expressed as

$$
\Delta \varepsilon(t, \tau)=\frac{\sigma_{0}(\tau)}{E_{c}(\tau)} \varphi(t, \tau)+\frac{\sigma(t)-\sigma_{0}(\tau)}{E_{c}(\tau)}[1+\chi(t, \tau) \varphi(t, \tau)] .
$$

Based on deformation compatibility and force equilibrium, one can obtain that

$$
\begin{array}{r}
\Delta N_{s}+\Delta N_{c}=0 . \\
\Delta N_{s}=\Delta \varepsilon(t, \tau) E_{s} A_{s} .
\end{array}
$$

where $\Delta N_{s}$ is the load increment of reinforcement; $\Delta N_{c}$ is the load increment of concrete; $E_{s}$ is the elastic modulus of reinforcement; $A_{s}$ is the cross section area of reinforcement. 
Substituting Eqs.9and 10 into8, the strain increment of reinforced concrete member can be rewritten as

$$
\begin{aligned}
& \Delta \varepsilon(t, \tau)=\frac{\sigma_{0}(\tau)}{E_{c}(\tau)} \varphi(t, \tau) /\left\{1+\frac{E_{s} A_{s}}{E_{c}(\tau) A_{c}}\right. {[1+\chi(t, \tau) \varphi(t, \tau)]\} . } \\
& \lambda=1 /\left\{1+E_{s} A_{s}[1+\chi(t, \tau) \varphi(t, \tau)] / E_{c}(\tau) A_{c}\right\} .
\end{aligned}
$$

Eq. 11 shows the effect of reinforcement on concrete deformation : the numerator termrepresents creep deformation of plain concrete; the denominator term represents the effect of reinforcement, which contains factors, such as loading age, concrete age, reinforcement ratio and can be used to evaluate the effect of reinforcement on concrete creep deformation.

Wang et al.[8] suggested the following ageing coefficient $\chi(t, \tau)$ equation based on creep constitutive theory and elastic continuation and plastic flow theory

$$
\chi(t, \tau)=\frac{1}{1-e^{\left[-0.665 \varphi(t, \tau)-0.107\left(1-e^{-3.131 \varphi(t, \tau)}\right)\right]}}-\frac{1}{\varphi(t, \tau)} .
$$

Due to the good agreement between the value calculated by Eq.13 and the measured data, Eq. 13 is selected in this paper to calculate the age coefficient.

Based on Eqs. 9-13, the concrete restrained stress reduction can be quantitatively calculated and the following equation can be used to evaluate the effect of restraint degree on concrete restrained stress reduction

$$
\xi=\frac{\Delta \sigma}{\sigma_{c}}
$$

where $\Delta \sigma$ is the concrete restrained stress reduction; $\sigma_{c}$ is the cracking stress of concrete.

\section{Case study}

Material Parameters. The specific creep of concrete $C(t, \tau)$ takes the following expression [7].

$$
C(t, \tau)=\sum_{j=1}^{2}\left(f_{i}+g_{i} \tau^{-p_{i}}\right)\left[1-e^{-r_{i}(t-\tau)}\right] .
$$

where $\tau$ is the loading age and $t$ is the concrete age.

Creep parameters are given in Table 1.

Table 1 Parameters of specific creep

\begin{tabular}{ccccc}
\hline Parameter & $f_{i}\left(10^{-4}\right)$ & $g_{i}\left(10^{-4}\right)$ & $p_{i}$ & $\gamma_{i}$ \\
\hline $\mathrm{i}=1$ & 0.1045 & 0.9614 & 0.7 & 0.3 \\
$\mathrm{i}=2$ & 0.2360 & 0.4012 & 0.7 & 0.05 \\
\hline
\end{tabular}

Table 2 shows the material properties used for calculation of concrete restrained stress.

Table 2Material properties for calculation of concrete restrained stress

\begin{tabular}{lll}
\hline Parameter & Unit & Value \\
\hline Concrete temperature & ${ }^{\circ} \mathrm{C}$ & $55 \times(1-\exp (-2 \times(\mathrm{t})))$ \\
Concrete elastic modulus & $\mathrm{MPa}$ & $44000 \times(1-\exp (-0.4 \times(\mathrm{t}) \wedge 0.34))$ \\
Reinforcement elastic modulus & $\mathrm{MPa}$ & 200000 \\
Concrete splitting strength & $\mathrm{MPa}$ & $2.4 \times\left(0.9 \times \exp (1-\operatorname{sqrt}(28 / \mathrm{t}))^{\wedge} 0.5\right)$ \\
\hline
\end{tabular}

Results and discussions.Fig.3 shows concrete restrained stress under different restrained degrees(the cooling rate is $0.17^{\circ} \mathrm{C} / \mathrm{hr}$ ). Due to the proportional relationship between concrete restrained stress and restraint degree, all curves of concrete restrained stress reach the second zero stress at same age; however, the value of concrete restrained stress is larger with higher restrained degree and concrete are prone to failure at early age.

Fig. 4 shows the relationship between restraint degree and concrete restrained stress reduction ratio.For the case of cooling rate with $0.17^{\circ} \mathrm{C} / \mathrm{hr}$, due to the high restraint degree, the positive effect of reinforcement on reduction of concrete restrained stress is minor. If the reinforced concrete 
member is totally restrained (i.e. $\gamma_{R}=1.0$ ), no reduction of concrete restrained stress is generated. When the restraint degree becomes 0.5 , almost $24 \%$ of concrete restrained stress is transferred to reinforcement and the potential crack possibility of concrete is greatly lowered.

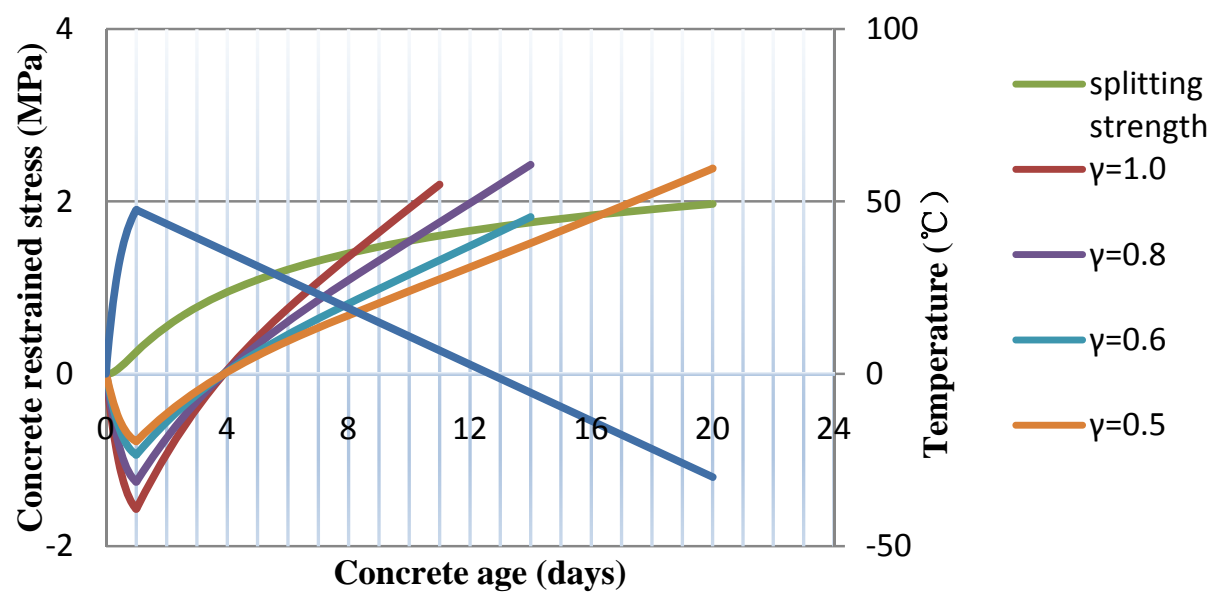

Fig.3 Evolution of concrete restrained stress with different restraint degrees

On the other hand, it also can be seen from Fig.4 that lower cooling rate is beneficial for reduction of concrete restrained stress. The concrete restrained stress reduction ratio under cooling rate of $0.17^{\circ} \mathrm{C} / \mathrm{hr}$ and $0.5^{\circ} \mathrm{C} / \mathrm{hr}$ is $24 \%$ and $17 \%$ respectively with a restraint degree of 0.5 .

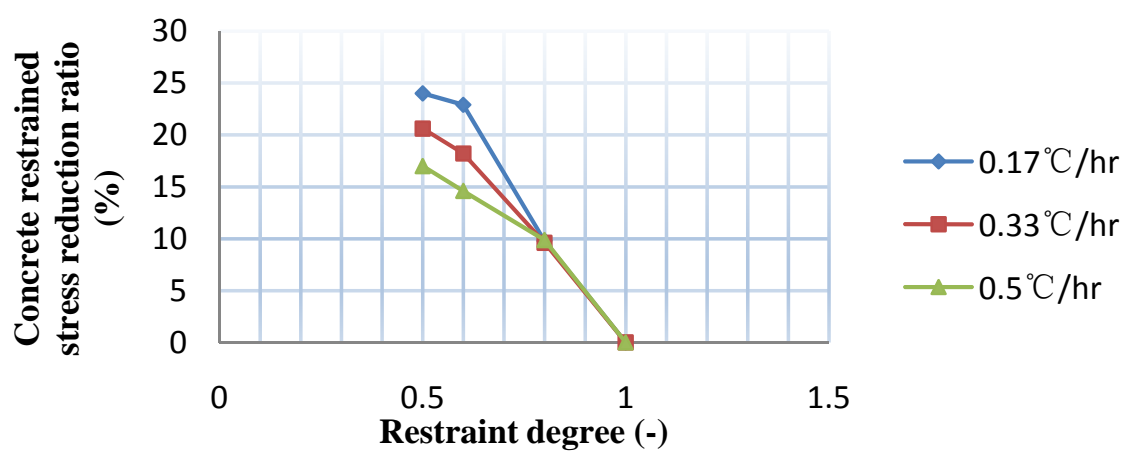

Fig.4 Relationship between restraint degree and concrete restrained stress reduction ratio

\section{Conclusions}

(1)Based on the age-adjusted effective modulus method, the effect of restraint degree and reinforcement on concrete restrained stress is quantitatively evaluated;

(2)The positive effect of reinforcement on reduction of concrete restrained stress is more predominant with lower restraint degree and slower cooling rate;

(3)The effect of reinforcement on reduction of concrete restrained stress is not a constant, but more considerable at early age and steady at later age.

\section{References}

[1]M. Larson: Thermal crack estimation in early age concrete( $\mathrm{PhD}$ thesis, Lulea University, Lulea, Sweden, 2003).

[2]G.X.Huang, R.Y.Hui, andX.J.Wang: Concrete creep and shrinkage(China electric power press, Beijing, China, 2011).

[3]W.Song, Y.Yuan and J. Gong: J. Southeast Univ.:Nat. Sci. Ed.Vol. 32 Sup.(2002), p.98-101.

[4]D. F.Cao, W. J.Ge, B. Y.Wang andY. M.Tu:Int. J. Civ. Eng. Vol. 13(2015), p.92-101.

[5]W. C.Xue, Y. M.Hu andW. Wang: China J. Highway Transportation. Vol. 21(2008), p.61-66.

[6]Z. P.Bazant: ACI J. Vol.69 (1972), p.212-217. 
[7]B.F.Zhu: Thermal stresses and temperature control of mass concrete (China Water and Power Press, Beijing, China,2009).

[8]X. W.Wang and J.Y.Pan:China Railway Sci. Vol. 17(1996), p.12-23. 